\title{
Product Recommendation in E-Commerce for Community center using Dependency algorithm
}

\author{
Spoorthi C, Dr. Pushpa Ravi Kumar, Arpitha C.N \\ Dept. of Computer Science and Engineering \\ Adichunchanagiri Institute of Technology \\ Chikmagalur-577102, Karnataka, India \\ E-mail: spoorthic25@gmail.com,flowersunpr@yahoo.co.in,arpithacn15@gmail.com
}

\begin{abstract}
Dependency algorithm have been broadly used to build a community recommendation system from the time when they distinguishes and shares the collective knowledge and occurrence. However it easily falls into the ambush of Mathew consequence, which have a tendency to recommend popular product and that's why the popular product item become increasingly less popular. Underneath this circumstance, the majority of the product items in the recommendation list are already common to customers and because of the performance would seriously decreases in finding exact item. To overcome this issue the prediction mold is built for shopping dataset. The online shopping dataset have been considered for cleaning using regex and mean weighted average vector is proposed. For selection of attributes the algorthim called PCA and Recursive feature elimination has been used and compared their accuracy. Dependency is proposed that can be recommended items to customer using a community build. Using the community mining techniques has been applied like degreeness, closeness, betweeness property of nodes is used to find the loyal customer in the community.
\end{abstract}

Keywords-Customer behaviour, E-Commerce, Feature extraction, Dependency, Community mining

\section{INTRODUCTION}

Everybody has their own informal community. Everybody has companions, families, and individuals they are familiar with. An online interpersonal interaction website basically makes our informal organizations unmistakable to other people who are not in our prompt system. So the absolute most significant element that recognizes an informal organization from a network is the manner by which individuals are held together on these destinations. In an informal organization, individuals are held together by pre-built up relational connections, for example, family relationship, companionship, cohorts, associates, colleagues, and so on. The associations are fabricated each one in turn. The essential reason that individuals join an interpersonal interaction site is to keep up former connections and set up new ones to grow their system. With this learning, it ought to be clear why Facebook, MySpace, and LinkedIn are informal organizations instead of community [1]. Community network is concerned much about significant amounts of customers acting together with some relationship. Network mining is one of the fundamental headings in casual association examination. The casual networks are different, heterogeneous and dynamic in nature. Which addresses a particular relationship reliant on some normally shared properties, and each kind of relationship may take an interest in a substitute occupation in a particular assignment [2].

Communities are held together by normal intrigue. It might be a diversion, something the network individuals are energetic around, a shared objective, a typical venture, or only the inclination for a comparable way of life, land area, or calling [3]. Plainly individuals join the network since they care about this basic intrigue that sticks the network individuals together. Some stay since they wanted to add to the reason; others come since they can profit by being a piece of the community. Data mining strategies is utilized for shopping dataset to recognize the devoted client and to give a more advantage, advancement and to deal with a network organize in an superior manner.

Community can be a major division between network definitions is whether vertices can have a place with a solitary network or any number of networks [3]. Avocations exist for each methodology, and eventually, the determination of which definition to utilize is likely space and application subordinate. For example, while investigating natural protein connection systems, if an examiner wishes to produce a proteins, a progressive disjoint technique is wanted. While breaking down interpersonal organizations, because of the assortment of affiliations and interests that an individual may have, a covering strategy might be increasingly fitting [4]. When the relationship among the customers is seen in an online business, it is unquestionably not difficult to predict the vitality among the clients. The principle point of this undertaking is to recognize the key player in a network. The people group is work for preprocessed information. By utilizing an algorthim considered 


\section{International Journal of Research in Advent Technology, Vol.7, No.5, May 2019 E-ISSN: 2321-9637 \\ Available online at www.ijrat.org}

reliance the network is assemble and mining methods are utilized to anticipate the key player in a system.

\section{LITERATURE SURVEY}

\section{Selection of attributes}

Varun E [1] has proposed data mining system is essential to consider buying behavior of the customers in Ecommerce stores. By thought of the productive customer the examination started on complete envisioning the getting conduct properties of customer. The data mining structure is uncommonly valuable for relationship to start the relationship of customer with various things.

\section{Multi-relational network}

Pushpa Ravikumar [2] has proposed detecting people group in systems is a profoundly helpful, exceedingly non-insignificant errand. In specific spaces, it is sensible to expect that network structure covers. This requires characterizing the key thoughts of what covering networks ought to resemble.

\section{Feature extraction using PCA}

Spoorthi C [3] has proposed the utilization of covering network structure can possibly help in the cognizance of fundamental procedures in an undeniably interconnected world Intuition and the experimental perceptions contained in this part recommend the affiliations contained inside such networks catch basic and important connections which are understood in the information. The field is a long way from full grown, and different inquiries have emerged all through research which stays open issues.

\section{Data mining techniques for community network}

R.V.Kulkarni [4] has proposed the community recognition calculations have would in general spotlight on static systems. In any case, certifiable information can possibly be very unique. Accordingly, new strategies should be proposed to deal with system ties with a transient component. One basic augmentation to the work portrayed in this content would be a sociologically grounded edge weight work.

\section{Mining in beverage marketing}

Ray I Chang [5] has proposed the aphorisms spread out in this part endeavor to satisfy that need, while in the meantime being as negligible as conceivable to take into consideration methodological and application explicit varieties. Organize trade is fundamental for mining experience supportability and achievement. The synthesis displays a reasonable relationship between framework sponsorship and supportability. Quantifiable estimations factors have been appeared to affect compose assertion.

\section{METHODOLOGY}

Community is build using data mining technique for identifying the key customer using shopping dataset is explained in the figure 1.

\section{Data Collection}

The data is downloaded from a shopping website. In this dataset some noise is present so for removing the two algorthim is used and compared their accuracy.

\section{A. Data Preprocessing}

In a data preprocessing the algorthim is used to clean the data using regex to remove commas and unwanted words, The another algorthim is mean weighted average vector is used to fill the missing values.

\section{B. Feature Extraction}

The attribute is selected after the data is cleaned to build a community. The data which is preprocessed is made to reduce dimension.

\section{Building a Community}

In this framework can be seen as the most central unsupervised learning issue close by as one another issue of this sort it comprehends how to discover a structure in a social event of unlabelled data appeared.

\section{Community Mining}

When the community is constructed the following stage is to locate the unwavering customer from a system. From each system the centrality a measure is determined to locate the key player in an entire community organize.

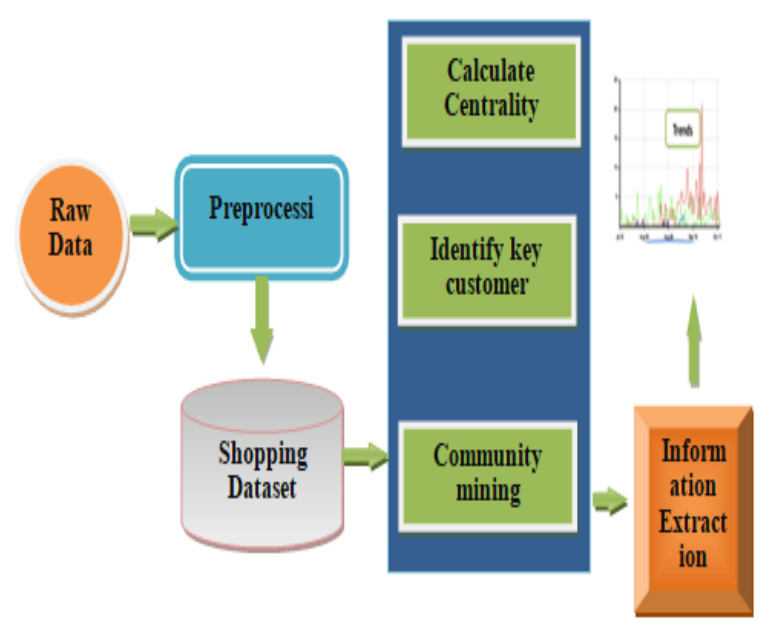

Figure 1: Community Mining for analyzing loyal customer behaviour 


\section{Algorithm: Recursive Feature Elimination}

1. Train the model on the training set

2. Calculate model performance

3. Calculate variable importance that is ranking

4. for Each subset size $S_{i p}, i=1 \ldots \ldots . . S$ do

5. Keep the $S_{\mathrm{i}}$ most important variable

6. [optional] pre-process the data

7. train the model on the training set using predictors

8. Calculate model performance

9. Recalculate the ranking for each predictor

10. end

11. Calculate the performance profile over the $S_{i}$

12. Determine the appropriate number of predictors

13. Use the model corresponding to the original $S_{i}$

\section{RESULT \& DISCUSSION}

The snapshot gives an idea of a proposed work. These are the portrayals taken when the system is adequately executing the code.

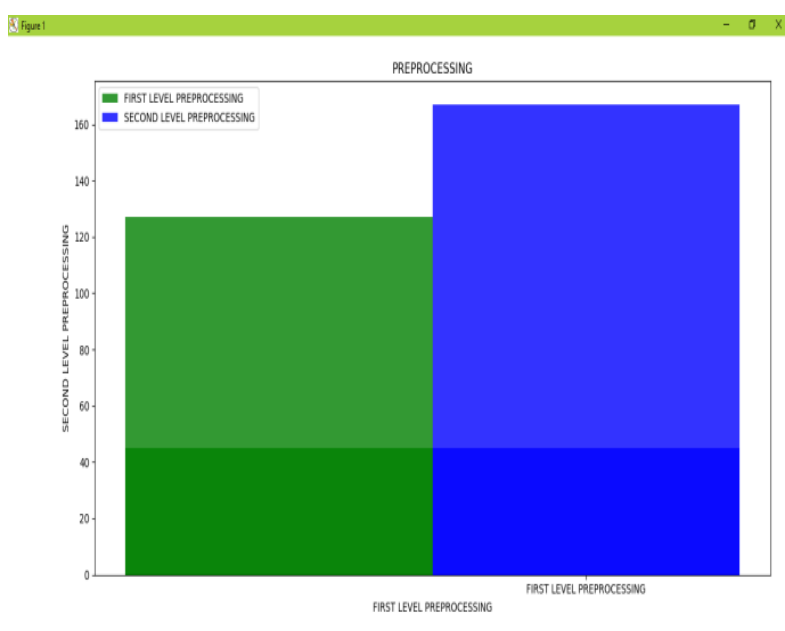

$A t \rightarrow+Q= \pm$

Figure 2: Raw data for preprocessing

Figure 2 portrays the before preprocessed shopping dataset which is taken from a known shopping sites. The information comprises of commotion and it is available in the arrangement exceed.

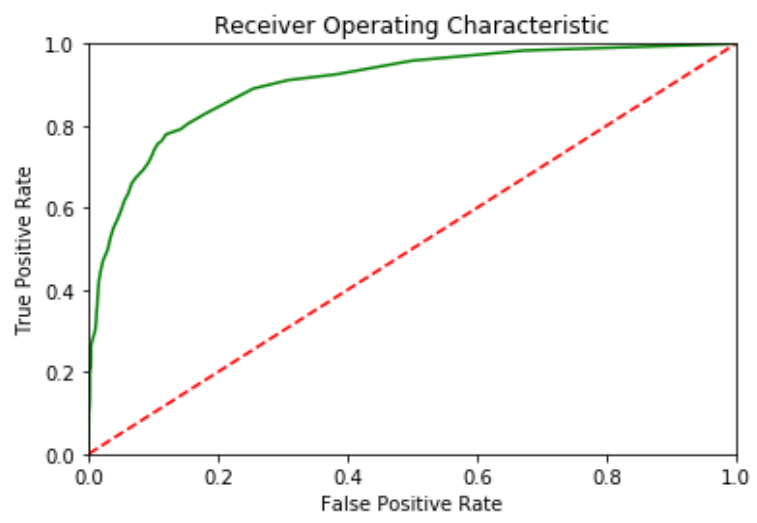

Figure 3: Pre-processed data for accuracy measure

Figure 3 gives the measure to pre-handled information to discover for exactness in a cleaning procedure. The perplexity network is likewise worked under ROC bend. The exactness desired preprocessed information is $91.89 \%$. The $\mathrm{X}$-hub indicates the bogus positive rate and $\mathrm{Y}$-hub determines the genuine positive rate.

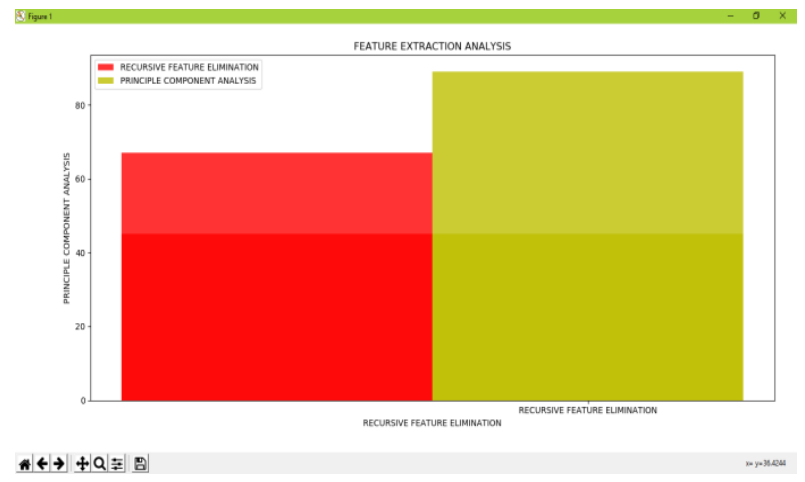

Figure 4: Feature extraction analysis graph

Figure 4 depicts the preprocessed information diagram which is loaded up with missing qualities. By applying the algorthim of highlight extraction it gives the significant properties for network assemble

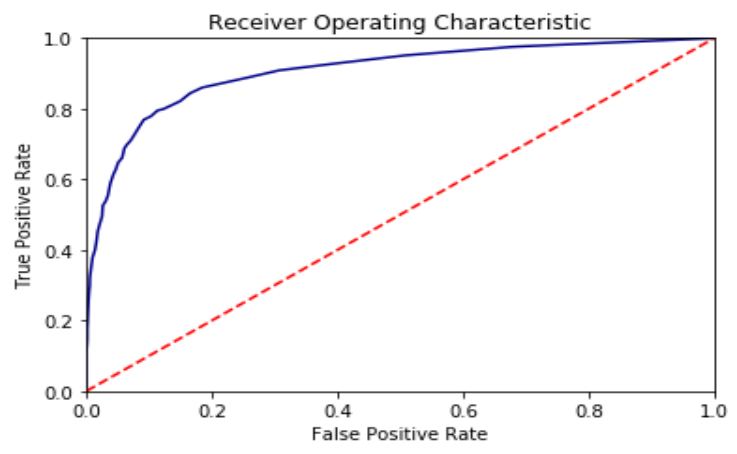

Figure 5: Feature extraction analysis graph 


\section{International Journal of Research in Advent Technology, Vol.7, No.5, May 2019 E-ISSN: 2321-9637 \\ Available online at www.ijrat.org}

Figure 5 gives a concise portrayal about the element choice procedure. The exactness for anticipating the dedicated client in an E-business utilizing a disarray grid. The ROC bend gives the depiction about the $\mathrm{X}$-pivot gives the bogus positive and Y-hub determines the genuine positive with exactness $92.25 \%$.

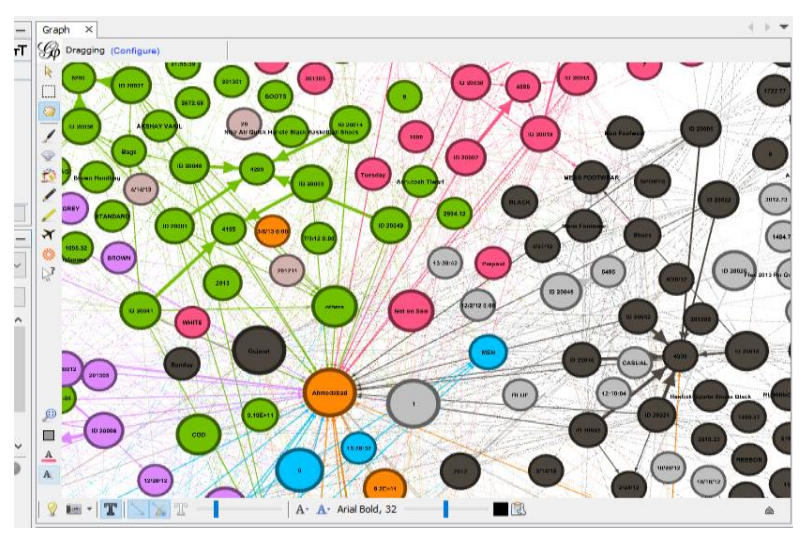

Figure 6: Community network formation

Figure 6 present the community network formation with a different category. It gives brief description about each network and formation.

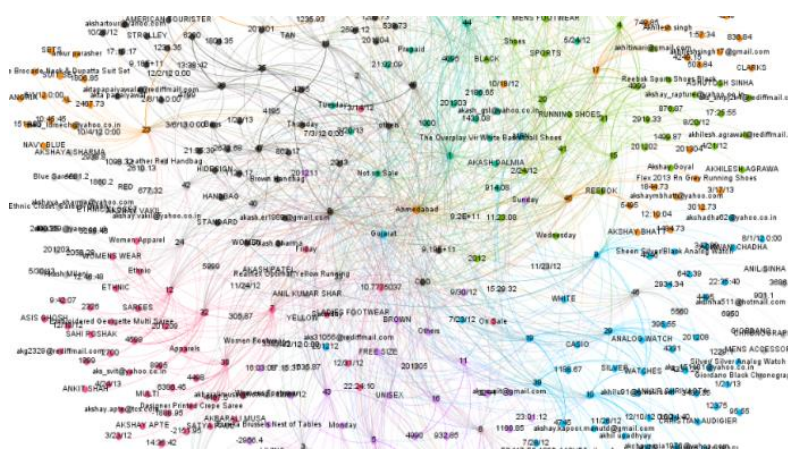

Figure 7: Community building for each network

Figure 7 present the measure of a hub and edges with hues. It is determining the every network in a little system. The algorthim measured quality is utilized for structure a system.

Figure 8 gives the closeness centrality an estimation of a degree, closeness, and betweeness to ascertain and to average these three qualities to discover the network.

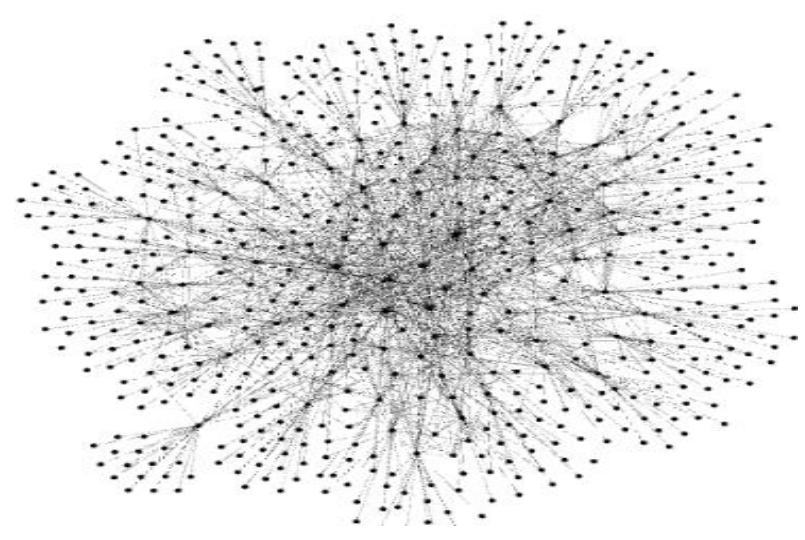

Figure 8: Centrality measure for community

Figure 9 depicting the key client from a count of a centrality measure utilizing a network mining systems. The people group is mined with a key player who get more advantages from a proprietor of a shop.

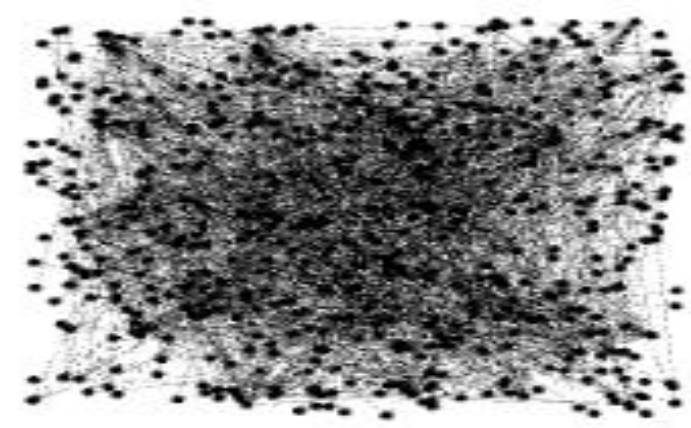

Figure 9: Calculating betweeness, closeness, degreeness for loyal customer identification

\section{CONCLUSION}

The customer direct can be poor down using the system collected using the shopping dataset. The preprocessing system regex and mean weighted ordinary vector removes highlight marks, displace invalid a motivation with legitimate regard exclusively. Feature assurance estimation PCA is found to give better accuracy of $90 \%$ when stood out from recursive component end methodology which gives the precision 69\%. Exactness and confusion framework of a pre-dealt with data is $91.89 \%$. The precision for feature isolated data is $92.25 \%$. The centrality measure is used to perceive the key customer in a shopping dataset which extends the pay of retailers or associations. The society is gathered using the dataset and all of the key players in the system recognized. The centrality measure can be also used in different zones to perceive the key player. In this proposed work the key player ID using information mining is seen to be logically capable. 


\section{ACKNOWLEDGMENTS}

I am highly thankful to my HOD Dr. Pushpa Ravikumar B.E., M.Tech., Ph. D., LMISTE Professor \& Head of the department of CS\&E, Adichunchanagiri Institute of Technology, Chikkmagaluru-577102 for her consistent support \& guidance all through the paper for her help regarding the usage of Product Recommendation in ECommerce for Community center using Dependency algorthim \& its aspects. Last but not the least in would like to thank all those who directly or indirectly assisted in the successful completion of the paper.

\section{REFERENCES}

[1] Varun E and Dr. Pushpa Ravikumar, "Attribute Selection for Telecommunication Churn Prediction", International Journal of Engineering \& Technology, Vol. 7, No 4.39, 2018, pp.506-509.

[2] Varun E and Dr. Pushpa Ravikumar, "Community Mining In Multi-Relational and Heterogeneous Telecom Network", IEEE 6th International Conference on Advanced Computing (IACC-2016), DOI:10.1109/IACC.2016.15.

[3] Spoorthi C, Dr Pushpa Ravikumar "Comparative Analysis of PCA and Recursive Feature Elimination Technique for Feature Extraction in Community Mining using E-commerce", International Journal of Engineering Research \& Technology (IJERT) - Vol. 8, Issue 05, page No 154 - 157, May 2019.

[4] Abhijit Raoranel, R.V.Kulkarni, "Data mining techniques: A Source for Consumer Behaviour Analysis", International Journal of Database Management System (IJDM), vol 2, August 2010.

[5] Chun Fu Lin, Yu Hsin Hung, and Ray I Chang, "Mining Customer Behavior Knowledge to Develop Analytical Expert System for Beverage Marketing", International Journal of Computer Trends and Technology (IJCTT) - volume 4 Issue 4 -April 2013.

[6] Bhumika Pahwa, Dr. S. Taruna, Neeti Kasliwal, "Role of Data mining in analyzing consumer's online buying behaviour", International Journal of Business and Management Invention, volume 6, Issue 11, page No 45-51, November 2017.

[7] Afolabi Ibukun.T, Olufunke Oladipupo, Rowland E. Worlu \& Akinyemi I. O, "A Systematic Review of Consumer Behaviour Prediction Studies", Covenant Journal of Business \& Social Sciences (CJBSS), Vol. 7, No.1, June 2016.

[8] Chun Fu Lin, Yu Hsin Hung, and Ray I Chang, "Mining Customer Behavior Knowledge to Develop Analytical Expert System for Beverage Marketing", International Journal of Computer
Trends and Technology (IJCTT) - volume4Issue4 -April 2013.

[9] Sisi Que, Kwame Awuah-Offei, "Framework for mining community consultation based on discrete choice theory", International Journal Mining and Mineral Engineering, Vol. 5, No. 1, 2014.

[10] Sandeep Kumar, Rakesh Kumar Arora, "Analyzing Customer Behaviour through Data Mining", International Journal of Computer Applications Technology and Research, Volume 4, Issue 12, page no 884 - 888, 2015.

[11] Milan Patel, Srushti Karvekar, Zeal Mehta, "Customer Behaviour Model Using Data Mining", International Journal of Advanced Technology in Engineering and Science, Volume No.02, Special Issue No. 01, September 2014.

[12] Dattatray V. Bhate, M. Yaseen Pasha, "Analyzing Target Customer Behavior Using Data Mining Techniques for E- Commerce Data", International Journal of Innovative Research in Computer Science \& Technology (IJIRCST) page no.2347-5552, Volume-2, Issue-1, January 2014

[13] Felipe Grando, Lisandro Z Granville, Luis C. Lamb, "Machine Learning in Network Centrality Measures: Tutorial and Outlook", ACM Computing Surveys, vol 51(5), article 102, 2018. 